\title{
Estudio piloto de detección de parásitos en frutas y hortalizas expendidas en los mercados públicos y privados de la ciudad de Bogota D.C.
}

\author{
Nelsi Andrea_Camargo Castillo, Silvia Campuzano. \\ Universidad Colegio Mayor de Cundinamarca, Programa de Bacteriología, Bogotá, Cundinamarca. \\ Correspondencia: nelcyta@yahoo.es.
}

Recibido: 23-03-2006 / Aceptado:11-05-2006

\section{Resumen}

Las enfermedades transmitidas por los alimentos contaminados, representan un grave problema de salúd pública, muchos microorganismos son capaces de sobrevivir a condiciones adversas para su desarrollo, por esta razón pueden encontrarse en las frutas y vegetales crudos.

Se pretende con este trabajo, exaltar la importancia de validar e implementar los métodos para detectar parásitos en frutas y hortalizas durante el proceso de las buenas prácticas agrícolas, ya que algunos de estos productos por sus características físicas están más expuestos a contaminación y por lo tanto son portadores de agentes causales de enfermedades.

El propósito fue recolectar muestras de frutas y hortalizas de los diferentes puntos de venta, y someterlos a estudio tomando un método de diagnóstico usado por otros autores para el aislamiento de parásitos en este tipo de muestras, una vez procesadas en el laboratorio se identificaron los agentes causales más comunes de los trastornos intestinales. Con los resultados de este estudio, se determinaron los parásitos encontrados en frutas y verduras mediante análisis parasitológico y se compararon los dos productos: frutas y hortalizas en cuanto a la contaminación con parásitos, socializando los resultados con los distribuidores y proponiendo condiciones de prevención.

Adicionalmente, se evaluó la inocuidad de los puntos de venta, la calidad de sus servicios y los saneamientos que ofrecen para eliminar la contaminación, proyectando beneficiar a los consumidores y comercializadores de los productos agrícolas, para disminuir el riesgo de contaminación.

Palabras clave: contaminación, epidemiología, parásitos, frutas, verduras, Bogotá.

\footnotetext{
Abstract

The diseases transmitted by contaminated foods, they represent a serious problem of health publishes, many microorganisms are able to survive adverse conditions for their development, therefore the fruits and crude vegetables can be contaminated.

It is tried with this work, to raise the importance of validating and of implementing the methods to detect parasites in fruits and vegetables during the process of the good ones you practice agricultural, since some of these products by their physical characteristics more are exposed to contamination and therefore they are carrying of causal agents of diseases.
} 
NOVA - PUBLICACIÓN CIENTÍFICA ISSN:1794-2470 VOL.4 No. 5 ENERO - JUNIO DE 2006:1-116

The intention was to collect samples of fruits and vegetables from the different points of sale, and to put them for to study taking a method from diagnosis used by other authors for the isolation of parasites in this type of samples. Once they were processed in the laboratory, they were identified for the causal and common agents of the intestinal upheavals. With the results of this study, it was tried to determine the parasites found in fruits and vegetables by means of parasitological analysis, to compare products both: fruits and vegetables as far as the contamination with parasites, to socialize the results with the distributors proposing conditions of prevention.

In addition the points of sale was evaluated, the quality of its services and the cleanings that offer to eliminate the contamination, projecting to benefit to the consumers and drug dealers from agricultural products, in order to diminish the contamination risk.

Key words: contamination, epidemiologic, parasites, fruits, vegetables, Bogotá.

\section{Introducción}

El consumo de frutas y hortalizas es vital para la salud humana puesto que poseen innumerables propiedades alimenticias, son fuente Inagotable de vitaminas, minerales, fibra y energía. Sin embargo, por sus características físicas, algunos de estos productos están expuestos a contaminación de tipo biológico y químico, situación que genera un riesgo para la salud humana (1).

Al momento de la compra, las frutas y hortalizas aparte de parecer frescas y apetitosas, también deben estar libres de residuos químicos, hongos, parásitos o insectos, que aunque en ocasiones no destruyen los productos macroscópicamente sí perturban silenciosamente la salud del consumidor, que inocente de estos riesgos compra el producto sin mayor atención.

Las buenas prácticas agrícolas garantizan la obtención de frutos de alta calidad, la protección del medio ambiente, la salud de los trabajadores y la inocuidad de los productos agrícolas (2-4). Sin embargo, se presentan casos de contaminación producidos por el uso de agua de riego contaminada con heces fecales de humanos y animales, por los procesos inadecuados en los campos de cultivo, prácticas deficientes de desinfección, condiciones inapropiadas durante el empaque, higiene deficiente de los trabajadores y el mal manejo durante su almacenamiento, estos alimentos son transportados directamente desde los cultivos a los puntos de distribución donde el consumidor los compra de manera libre, siendo llevados a los hogares donde no son lavados de manera adecuada generando de esta forma que los alimentos se conviertan en un riesgo para la población(3,6-9).

Las parasitosis intestinales son una de las consecuencias de la ingesta de estos productos, por este motivo se deben mejorar las prácticas agrícolas al momento de la distribución de los productos frutihoticolas a las ciudades y exigir estas pruebas a los comercializadores para disminuir el riesgo (10-13).

Una vez vendido el producto se hace muy difícil garantizar la manipulación adecuada y los riesgos epidemiológicos solamente irían dirigidos hacia el manejo de los alimentos en el hogar cerrando el ciclo con la concientización de la comunidad del buen lavado y buena cocción de los alimentos.

La identificación de parásitos en frutas y hortalizas es una forma de disminuir y prevenir las parasitosis causadas por los alimentos contaminados $(14,15)$, por esta razón vigilando los puntos críticos de los procesos de producción y buscando la forma de removerlos antes de que el producto salga a la venta o antes del consumo son estrategias de prevención de este tipo de infección. Sin embargo, los métodos conocidos para este proceso no están validados lo que hace 
que no se tenga registro de calidad y se conviertan en un problema de salud publica en Colombia (16).

De esta forma, es necesario validar los métodos de detección de parásitos para controlar los puntos críticos de las prácticas agrícolas y lograrlos corregir a tiempo garantizando que las frutas y hortalizas sean aptas para el consumo humano (6-8)

\section{Materiales y métodos}

\section{Selección de puntos de toma de muestra}

Se realizó un sondeo en puntos de venta de los productos agrícolas en la ciudad de Bogotá. Para el proyecto se tomaron 10 puntos de venta más populares, informados por la Alcaldía como los más concurridos por los capitalinos (5 públicos y 5 privados).

Al momento de la toma de muestra se realizó una conferencia en la que se explicó a los comerciantes los objetivos del proyecto haciendo énfasis en las buenas prácticas agrícolas y la importancia de su aplicación.

El numero total de muestras colectadas fue de 100.

\section{Selección y obtención de la muestra}

La toma y procesamiento de las muestras se realizó en el transcurso de 24 días, en los cuales se asistió a los puntos de venta cada tercer día. Para la obtención de la muestra se colectaron $40 \mathrm{~g}$ de 5 hortalizas diferentes como lechuga, tallos, acelga, apio y espinaca y 5 frutas: mora, lulo, uvas, guayaba y mango dulce las cuales se encuentran en casi todos los puntos de venta, estos productos se encontraban frescos y en perfecto estado macroscópico al momento del muestreo.

Cada producto se empacó en las bolsas entregadas por el supermercado para evitar el contacto entre las mismas, cada bolsa fue marcada con el número consecutivo de estudio y para cada muestra se registro el formato de recolección el cual tiene información pertinente para datos epidemiológicos.

www.unicolmayor.edu.co

\section{Procesamiento de las muestras}

Las muestras fueron trasladadas inmediatamente a los laboratorios de la Universidad Colegio Mayor de Cundinamarca donde se realizó el procedimiento para detectar los parásitos.

Las muestras se procesaron según la técnica de Álvarez modificada referenciada en el artículo realizado por Luis Travieso (4).

\section{Procedimiento}

Se deshojaron y partieron en trozos los $40 \mathrm{~g}$ de muestra. Cada producto se introdujo en un vaso de precipitado limpio con capacidad para $500 \mathrm{ml}$ que contenía $400 \mathrm{ml}$ de agua destilada (este fue marcado con el número consecutivo de cada muestra), se agitó el contenido fuertemente y se dejó en reposo por 24 horas.

Trascurridas las 24 horas se retiraron las muestras dejando el agua en reposo una hora mas, posteriormente se decantó 9/10 partes de la solución.

El sedimento fue colocado en tubos, los cuales se centrifugaron por 10 minutos a $3000 \mathrm{rpm}$, luego se descartó el sobrenadante para obtener la muestra final para la lectura.

La lectura se realizó colocando la muestra sobre una gota de solución salina al $0.85 \%$ y una gota de lugol observándolas al microscopio con los objetivos de $10 \mathrm{X}$ y $40 \mathrm{X}$.

\section{Resultados}

El estudio demostró la presencia de parásitos intestinales en un $48 \%$. De este resultado el $80 \%$ de positividad se encontró en las hortalizas y el $20 \%$ restante se halló en las frutas.

En cuanto a la inocuidad de los productos en los mercados públicos y privados se demostró que el 52\% de los parásitos se encontró en los establecimientos privados y el $48 \%$ restante se obtuvo de los establecimientos públicos.

El análisis arrojo resultados en los que se aprecian los parásitos distribuidos de la siguiente manera: 


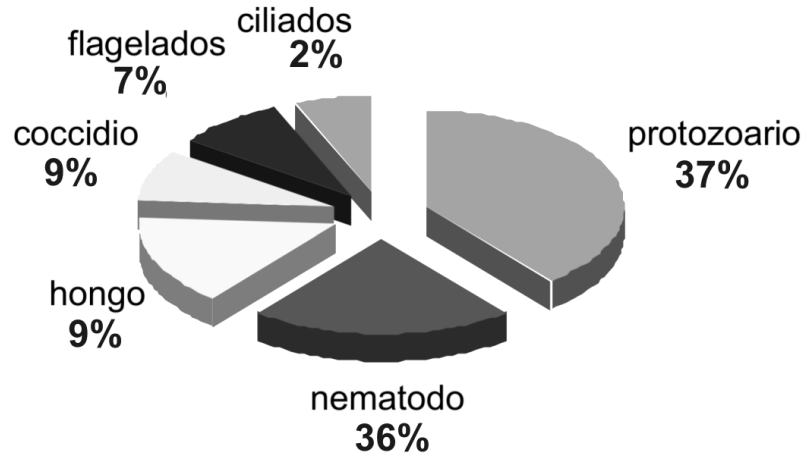

Figura 1. Distribución porcentual de parásitos encontrados.

protozoarios con el $37 \%$, nematodos con un $36 \%$, hongos en un $9 \%$, coccidios en un $9 \%$, flagelados en un $7 \%$ y ciliados en un $2 \%$, como se presenta en la Figura 1.

En cuanto a morfología el análisis mostró parásitos en los siguientes estados evolutivos, el $38 \%$ de las muestras positivas presentaron quistes, el $22 \%$ huevos, el 15\% larvas, 9\% de ooquistes, $9 \%$ de levaduras y un $7 \%$ de trofozoitos.
En el análisis consolidado se encontró la siguiente distribución por especie, Figura 3.

\section{Discusión}

La evidente presencia de parásitos intestinales en los alimentos analizados refuerza el tema en discusión como es la falencia que se presenta en los procesos agrícolas y la mala manipulación de los productos en los puntos de distribución, dado que este tipo de productos es de fácil acceso y son productos de consumo masivo por sus propiedades alimenticias, la vulnerabilidad de la población es más alta y la propensión al contagio de enfermedades de este tipo es alta, y así se convierte en un problema de salud publica. Los parásitos detectados son causantes de trastornos intestinales principalmente en la población infantil, personas de la tercera edad y pacientes inmunocomprometidos (5).
Entamoeba coli

Strongyloides stercoralis

Uncinarias

Entamoeba histolytica

levaduras

Blastocystis hominis

Giardia Iamblia

Toxocara canis

Enterobius vermicularis

Áscaris lumbricoides

Balantidium coli
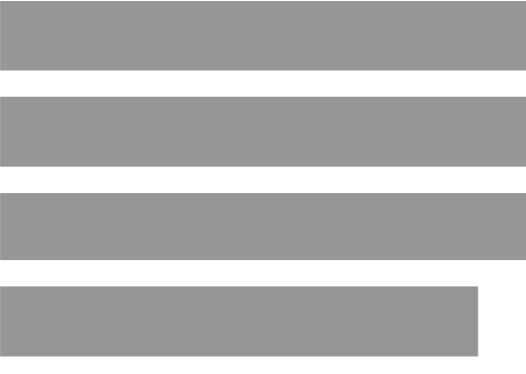

$13 \%$
$15 \%$

$15 \%$
$9 \%$

$9 \%$
$7 \%$
$2 \%$

$2 \%$

$2 \%$

$2 \%$

Figura 2. Consolidado por especies 


\section{El presente estudio permitió identificar claramen-} te el grado de contaminación de los productos alimenticios, encontrando como principales agentes causales a nematodos y protozoarios. Adicionalmente mostró que las condiciones provistas en los diferentes puntos de distribución ya fueran plazas de mercado o almacenes de cadena no presentan diferencias sustanciales en cuanto al manejo de los productos, por lo tanto no se determinaron diferencias en el tipo de parásitos detectados.

Debido a los hallazgos realizados se hace necesaria la concientización de la población acerca de la importancia de la higiene en el manejo y manipulación de los alimentos y se comprueba la importancia de implementar técnicas de diagnóstico parasitológico en los procesos de buenas practicas agrícolas, en plazas de mercado y almacenes de cadena.

\section{Referencias}

1. Chaidez C. Inocuidad de frutas y hortalizas frescas: Efecto del agua contaminada. Agua Latinoamérica. 2002; 2(3).

2. Acuerdo de Cartagena. Normas y procedimientos reglamentarios de la industria de alimentos. ANDI. Ley 9a de 1979. $331-332 p$.

3. Dirección General de Inocuidad Agroalimentaria, Acuícola y Pesquera Manual de Almacenamiento y Transporte de Frutas y Hortalizas Frescas en Materia de Inocuidad, México 6p.
4. Traviezo L, Davila J, Rodríguez $\mathrm{R}$, et al. Contaminación enteroparasitaria de lechugas expendidas en mercados del estado Lara. Venezuela. Parasitol. Latinoam. Jul. 2004; 59 (3):167-170.

5. Gómez J. Parasitosis intestinales frecuentes. Protocolos diagnósticos y terapéuticos en pediatría.

6. Cartaya Z, Mendoza C, Oyon R. Presencia de Entamoeba histolytica, ascaris lumbricoides y coliformes totales en ensaladas para perrocalientes, expendidas en el centro de la ciudad de Maracay. Rev. Soc. Ven. Microbiol., ene. 2003; 23 (1): 60-64

7. Tananta V, Chávez A, Casas E. Presencia de enteroparásitos en lechuga (Lactuca sativa) en establecimientos de consumo público de alimentos en el cercado de Lima. Rev. Inv. Vet Peru 2004; 15 (2):157-162.

8. Mejorando la Seguridad y Calidad de Frutas y Hortalizas Frescas: Manual de Formación para Instructores. Copyright (C) 2002 University of Maryland.

9. Bailenguer J. Mechanisms of parasitological concentration in coprology and their practical consequences. Journal of American Medical Technology. 1979;41: 65-71.

10. Pierangeli N, Giayetto A. Estacionalidad de parásitos intestinales en suelos periurbanos de la ciudad de Neuquén, Patagonia, Argentina. Tropical Medicine and International Health. 2003; 8 (3) 259-263.

11. Oficina regional de la Fao para América Latina y el Caribe. Enero 2004, Organización de las Naciones Unidas para la agricultura y la alimentación las buenas prácticas agrícolas. Pág.4.

12. Lau Chong C. Evaluación de técnicas parasicológicas en el diagnostico de estrongiloidiasis por Strongiloides stercolaris; Rev. Med Hered. 2005;16 (1).

13. Flores M, Marcos Luis, Terashima I, et al. Fas2-ELISA y la técnica de sedimentación rápida modificada por lumbreras en el diagnóstico de la infección por Fasciola hepática. Rev Med Hered. 2002; 13 (2): 49-57.

14. Abramovich B. Enteroparásitos Potencialidad de su transmisión a través del agua Facultad de Bioquímica y Cs. Biológicas. U.N.L.2000.

15. Ruiz O. Atlas de parasitología, estructuras parasitarias presentes en las aguas residuales. Universidad de Pamplona 11- 18p.

16. Ministerio de Salud. Anexo 4.33 resolución numero 14712 de 1984 